PROCEEDINGS OF THE

AMERICAN MATHEMATICAL SOCIETY

Volume 126, Number 2, February 1998, Pages 569-576

S 0002-9939(98)04105-7

\title{
SOME HARMONIC $n$-SLIT MAPPINGS
}

\author{
MICHAEL J. DORFF
}

(Communicated by Albert Baernstein II)

\begin{abstract}
The class $S_{H}$ consists of univalent, harmonic, and sense-preserving functions $f$ in the unit disk, $\Delta$, such that $f=h+\bar{g}$ where $h(z)=z+\sum_{2}^{\infty} a_{k} z^{k}$, $g(z)=\sum_{1}^{\infty} b_{k} z^{k} . S_{H}^{O}$ will denote the subclass with $b_{1}=0$. We present a collection of $n$-slit mappings $(n \geq 2)$ and prove that the 2-slit mappings are in $S_{H}$ while for $n \geq 3$ the mappings are in $S_{H}^{O}$. Finally we show that these mappings establish the sharpness of a previous theorem by Clunie and SheilSmall while disproving a conjecture about the inner mapping radius.
\end{abstract}

\section{INTRODUCTION}

A continuous function $f=u+i v$ is a complex-valued, harmonic function if both $u$ and $v$ are real harmonic. Throughout this paper we will discuss harmonic functions that are univalent and sense-preserving on $\Delta=\{z:|z|<1\}$. Clunie and SheilSmall [2] showed that such a mapping can be written in the form $f=h+\bar{g}$, where $h$ and $g$ are analytic and $\left|h^{\prime}(z)\right|>\left|g^{\prime}(z)\right|$. Hence $f(z)=\sum_{k=0}^{\infty} a_{k} z^{k}+\sum_{k=1}^{\infty} b_{k} \bar{z}^{k}$. Let $S_{H}$ be the class of such functions for which $a_{1}=1$ and $a_{0}=0$, and let $S_{H}^{O}$ be the subset of $S_{H}$ in which $b_{1}=0$. Note that the familiar class $S$ of analytic univalent functions is contained in $S_{H}^{O}$.

Clunie and Sheil-Small provided a method for constructing harmonic univalent functions for which $f(\Delta)$ is convex in the direction of the real axis. A domain $\Omega$ is convex in the direction of the real axis if every line parallel to the real axis has a connected intersection with $\Omega$. Using their method examples of harmonic 1-slit mappings can be found, and it is believed that certain extremal properties are attained among these functions [1]. Their approach makes it also possible to construct examples of harmonic 2-slit mappings. However, it seems that besides the standard analytic functions, no one has constructed examples of harmonic symmetric $n$-slit mappings, for $n \geq 3$.

In their paper, Clunie and Sheil-Small also explored the effect of extending the class $S$ to $S_{H}^{O}$ and $S_{H}$. In one instance, they considered $R(f)=\min \{|w|: w \notin f(\Delta)\}$. They showed that if $f \in S_{H}$, then $0<R(f) \leq \frac{2 \pi \sqrt{6}}{9}<1.72$. For $f \in S_{H}^{O}$ they proved that the corresponding value, $R_{O}(f)$, satisfies $\frac{1}{16} \leq R_{O}(f) \leq \frac{2 \pi \sqrt{3}}{9}<1.21$. Recall that for the class $S$, the lower bound is $\frac{1}{4}$ and the upper bound is 1 . By letting

Received by the editors April 19, 1996 and, in revised form, August 23, 1996.

1991 Mathematics Subject Classification. Primary 30C55, 30C45.

This work represents part the author's Ph.D. thesis at the University of Kentucky.

(c)1998 American Mathematical Society 
TABLE 1

\begin{tabular}{||c|c|l|c||}
\hline & Known facts & Conjectured & Our construction \\
\hline \hline$f \in S$ & $\frac{1}{4} \leq R_{O}(f) \leq 1$ & & \\
\hline$f \in S_{H}^{O}$ & $\frac{1}{16} \leq R_{O}(f) \leq \frac{2 \pi \sqrt{3}}{9}$ & $\frac{1}{6} \leq R_{O}(f)$ & $f$ with $R_{O}(f)=\frac{2 \pi \sqrt{3}}{9}-\epsilon$ \\
\hline$f \in S_{H}$ & $0<R(f) \leq \frac{\pi}{2}$ & & $f$ with $R(f)=\frac{\pi}{2}-\epsilon$ \\
\hline
\end{tabular}

TABLE 2

\begin{tabular}{||c|c|c|c||}
\hline & Known facts & Conjectured & Our construction \\
\hline \hline$f \in S$ & $\rho_{O}(f)=1$ & & \\
\hline$f \in S_{H}^{O}$ & $\frac{1}{4} \leq \rho_{O}(f) \leq \frac{8 \pi \sqrt{3}}{9}$ & $\frac{2}{3} \leq \rho_{O}(f)$ & $f$ with $\rho_{O}(f)=\frac{2^{5 / 3} \pi \sqrt{3}}{9}-\epsilon$ \\
\hline$f \in S_{H}$ & $0<\rho(f) \leq 2 \pi$ & $\rho(f) \leq \frac{\pi}{2}$ & $f$ with $\rho(f)=\pi-\epsilon$ \\
\hline
\end{tabular}

$f(z)=z+\alpha \bar{z}$ for $|\alpha|<1$, we see that the bound $0<R(f)$ is sharp. Also Clunie and Sheil-Small conjectured that $\frac{1}{6} \leq R_{O}(f)$. At the time of the paper it was not known whether the upper bounds for $R(f)$ and $R_{O}(f)$ were the best possible. A year later, Hall [5] showed that the upper bound for $R(f)$ can be decreased to $\frac{\pi}{2} \approx 1.57$. He established that this is the best possible constant by providing an example of a function that is the limit of mappings in $S_{H}$ which take $\Delta$ onto concentric disks whose radii approach $\frac{\pi}{2}$. See Table 1 for a summary of these known facts and conjectures.

In a separate paper [6], Sheil-Small discussed the inner mapping radius, $\rho(f)$, of the domain $f(\Delta)$ for $f \in S_{H}$. The inner mapping radius is defined as the real number $F^{\prime}(0)$, where $F(z)$ is the analytic function that maps $\Delta$ onto $f(\Delta)$ and satisfies the conditions $F(0)=0, F^{\prime}(0)>0$. If $f \in S_{H}^{O}$ then the inner mapping radius will be denoted by $\rho_{O}(f)$. Note that $\rho(f)$ cannot be larger than $2 \pi$, because of the Koebe $\frac{1}{4}$-theorem and Hall's result. Similarly, $\rho_{O}(f)$ is bounded above by $\frac{8 \pi \sqrt{3}}{9}<4.837$. Based upon Hall's example, Sheil-Small $[1,6]$ conjectured that $\rho(f) \leq \frac{\pi}{2}$. As far as we know no conjecture has been made on the upper bound for $\rho_{O}(f)$. Also, in [6], Sheil-Small proved that $\frac{1}{4} \leq \rho_{O}(f)$. Since $\rho_{O}\left(k_{O}\right)=\frac{2}{3}$, where $k_{O}$ is the proposed harmonic Koebe function, he conjectured that $\frac{2}{3} \leq \rho_{O}(f)$. See Table 2 .

In this paper we will present a collection of harmonic $n$-slit mappings, $f(z, n, s)$, for $n \geq 2$ and parametrized by $s$, where $0 \leq s<1$, in which the slits are symmetric about the origin and move away from the origin as $s$ increases. In this collection, there is a family of 2-slit mappings with each function, $f$, in $S_{H}$, such that $f(\Delta)$ will contain all the points in the disk whose radius approaches $\frac{\pi}{2}$ as $s$ approaches 1. Hence there are functions in this family for which the inner mapping radius can be made arbitrarily close to $\pi$. This provides a counterexample to Sheil-Small's conjecture. In addition, this collection contains a family of 3-slit mappings that are in $S_{H}^{O}$ and that will establish the value $R_{O}(f)=\frac{2 \pi \sqrt{3}}{9}$ obtained by Clunie and Sheil-Small as the best possible. This will also show that the inner mapping radius for $f \in S_{H}^{O}$ can be as large as $\frac{2^{5 / 3} \pi \sqrt{3}}{9}>1.91$. See Tables 1 and 2 . 


\section{A COLLECTION OF SYMMETRIC $n$-SLIT MAPPINGS}

For $z \in \Delta, n=2,3,4, \ldots$, and $0 \leq s<1$, consider the collection of functions

$$
\begin{aligned}
f(z, n, s) & =(1-s) f_{1}(z, n)+s f_{2}(z, n) \\
& =(1-s) \frac{z}{\left(1-z^{n}\right)^{2 / n}}+s\left(\frac{\pi}{n \sin \frac{\pi}{n}}\right) \frac{1}{2 \pi} \int_{0}^{2 \pi} \operatorname{Re}\left(\frac{1+z e^{-i t}}{1-z e^{-i t}}\right) e^{i \varphi(t)} d t
\end{aligned}
$$

where $\varphi(t)=\frac{\pi(2 k+1)}{n} \quad\left(\frac{2 \pi k}{n} \leq t<\frac{2 \pi(k+1)}{n}, k=0, \ldots, n-1\right)$. We will show that for $n=2, f \in S_{H}$ and it maps $\Delta$ onto the 2-slit domain with the tip of the slits at $\pm \frac{1-s+s \pi}{2}$ and the slits lying on the imaginary axis. See Figure 1. Similarly, for $n \geq 3$ we will demonstrate that $f \in S_{H}^{O}$ and $f(\Delta)$ is an $n$-slit domain whose slits start at points symmetrically placed on the circle of radius $(1-s) 4^{-1 / n}+s \pi /(n \sin (\pi / n))$ $(0 \leq s<1)$. See Figure 1 .

For each $n, f_{1}$ is an analytic function that maps $\Delta$ onto a $n$-slit domain that is symmetric with respect to the origin and to the real axis and where the slits start at the points $2^{-2 / n}\left(e^{i \pi(2 k+1) / n}\right)$ and form the angle $\frac{(2 k+1) \pi}{n}$ with the positive real axis, where $k=0, \ldots, n-1$. We know that

$$
f_{1}(z)=z \sum_{k=0}^{\infty}(-1)^{k}\left(\begin{array}{c}
k \\
-2 / n
\end{array}\right) z^{n k}
$$

where $\left(\begin{array}{c}k \\ -2 / n\end{array}\right)=1$ if $k=0$ and $\left(\begin{array}{c}k \\ -2 / n\end{array}\right)=\frac{(-2 / n)(-2 / n-1) \cdots(-2 / n-k+1)}{k !}$ otherwise.

Note that the function $f_{2}$ is the Poisson integral of boundary values concentrated at $e^{i \pi(2 k+1) / n}, k=0, \ldots, n-1$. In particular, for $n=2, f_{2}(\Delta)$ is a slit on the imaginary axis from $\frac{\pi}{2} i$ to $\frac{-\pi}{2} i$. For $n \geq 3, f_{2}$ is a harmonic function that maps $\Delta$ onto the region inside the regular $n$-gon whose vertices are at the points $(\pi /(n \sin (\pi / n))) e^{i \pi(2 k+1) / n}[4]$. Notice that $f_{2}$ can be written as

$$
\left(\frac{\pi}{n \sin \frac{\pi}{n}}\right) \sum_{k=0}^{n-1} \frac{e^{i \pi(2 k+1) / n}}{2 \pi} \int_{2 \pi k / n}^{2 \pi(k+1) / n} \operatorname{Re}\left(\frac{1+z e^{-i t}}{1-z e^{-i t}}\right) d t .
$$

Since $f_{2}$ is a harmonic function, $f_{2}=h_{2}+\overline{g_{2}}$, where $h_{2}, g_{2}$ are analytic functions in $\Delta$.

Each vertex of the polygonal region, $f_{2}(\Delta)$, lies on one of the slits of $f_{1}(\Delta)$.

The following lemma has been proved in [7]; we include our proof for completeness.

Lemma 2.1. Let $n \geq 2$. Then
1. $h_{2}(z)=\sum_{k=0}^{\infty} \frac{1}{k n+1} z^{k n+1}$ and $g_{2}(z)=\sum_{k=1}^{\infty} \frac{-1}{k n-1} z^{k n-1}$,
2. $h_{2}^{\prime}(z)=\frac{1}{1-z^{n}}$
3. $g_{2}^{\prime}(z)=\frac{-z^{n-2}}{1-z^{n}}$, and
4. $g_{2}^{\prime}(z)=-z^{n-2} h_{2}^{\prime}(z)$. 
Proof. To prove the first part, notice that

$$
\operatorname{Re}\left(\frac{1+z e^{-i t}}{1-z e^{-i t}}\right)=\operatorname{Re}\left[1+2 z e^{-i t}\left(\frac{1}{1-z e^{-i t}}\right)\right]=1+\sum_{k=1}^{\infty}\left(z^{k} e^{-i k t}+\bar{z}^{k} e^{i k t}\right) .
$$

The 2-slit apping

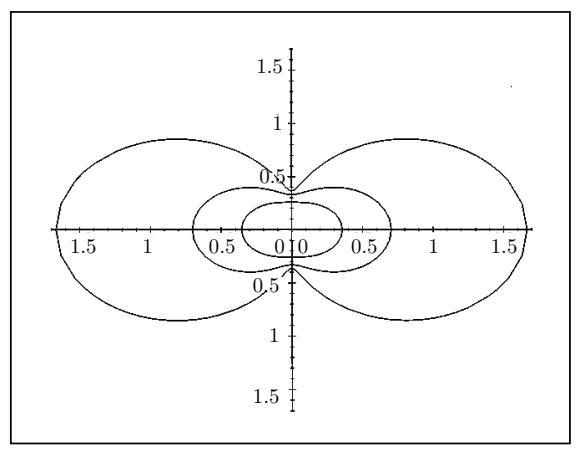

$s=14$

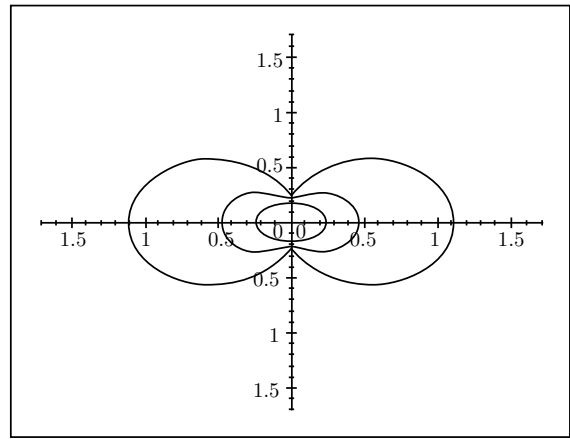

$s=12$

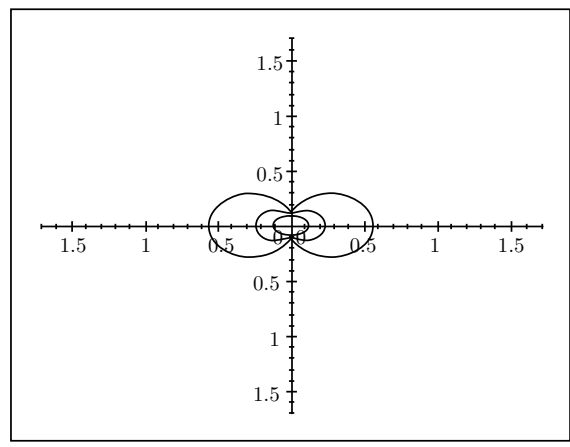

$s=34$
The 3-slit apping

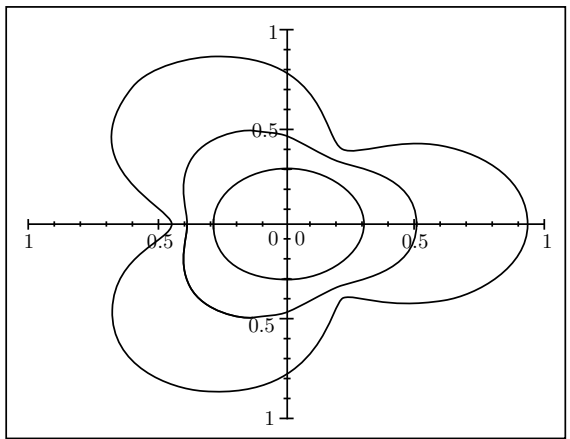

$s=14$

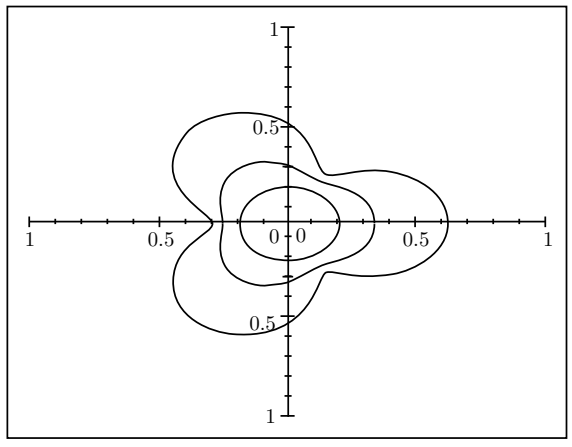

$s=12$

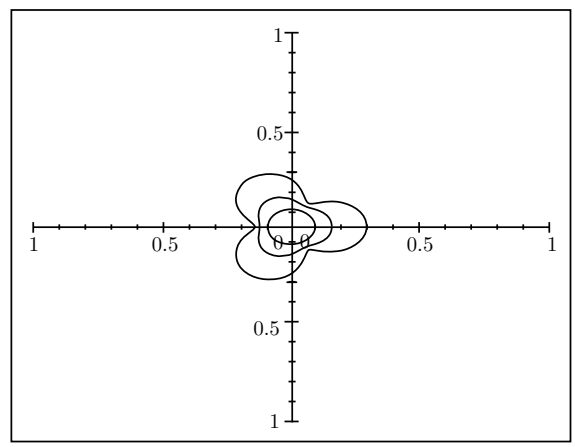

$s=34$

Figure 1. Image of circles of radius $\mathrm{r}=0.4,0.6$, and 0.8 . 
Hence by $(2)$

$$
f_{2}(z)=\left(\frac{\pi}{n \sin \frac{\pi}{n}}\right) \sum_{k=0}^{n-1} \frac{e^{i \pi(2 k+1) / n}}{2 \pi} \int_{2 \pi k / n}^{2 \pi(k+1) / n}\left[1+\sum_{j=1}^{\infty}\left(z^{j} e^{-i j t}+\bar{z}^{j} e^{i j t}\right)\right] d t .
$$

We have

$$
a_{0}=f_{2}(0)=\left(\frac{\pi}{n \sin \frac{\pi}{n}}\right) \frac{e^{i \pi / n}}{n} \sum_{k=0}^{n-1}\left(e^{i \pi 2 / n}\right)^{k}=0 .
$$

The coefficient of $z^{j}$, for $j \geq 1$, is

$$
\begin{aligned}
a_{j} & =\left(\frac{\pi}{n \sin \frac{\pi}{n}}\right) \sum_{k=0}^{n-1} \frac{e^{i \pi(2 k+1) / n}}{2 \pi} \int_{2 \pi k / n}^{2 \pi(k+1) / n} e^{-i j t} d t \\
& =\left(\frac{\sin \frac{j \pi}{n}}{j n \sin \frac{\pi}{n}}\right) e^{-i \pi(j-1) / n} \sum_{k=0}^{n-1}\left(e^{-i \pi 2(j-1) / n}\right)^{k} .
\end{aligned}
$$

Summing the geometric series we see that

$$
a_{j}= \begin{cases}\frac{1}{j} & \text { if } j=m n+1 \quad(m=0,1,2, \ldots), \\ 0 & \text { otherwise. }\end{cases}
$$

Similarly,

$$
b_{j}= \begin{cases}\frac{-1}{j} & \text { if } j=m n-1 \quad(m=1,2, \ldots), \\ 0 & \text { otherwise. }\end{cases}
$$

By differentiating the series for $h_{2}$ and $g_{2}$, we get the remaining three parts of the lemma.

Remark. These results about $f_{1}$ and $f_{2}$ provide us with some information about the function $f$. Recall that $f(z)=(1-s) f_{1}(z, n)+s f_{2}(z, n)$. Note that $f$ is harmonic and hence $f=h+\bar{g}=\sum_{k=0}^{\infty} a_{k} z^{k}+\sum_{k=1}^{\infty} b_{k} \bar{z}^{k}$. Let $s$ be fixed such that $0 \leq s<1$. It follows from (1), (3), (4), and (5) that if $n=2$, then the function $f$ has the coefficients $a_{0}=0, a_{1}=1$, and $b_{1}=-s$. If $n \geq 3$, the only change is that $b_{1}=0$.

Lemma 2.2. For any $n \geq 2, f$ is sense-preserving.

Proof. Clunie and Sheil-Small [2] showed that it is sufficient to verify that $\left|h^{\prime}(z)\right|=$ $\left|(1-s) \frac{1+z^{n}}{\left(1-z^{n}\right)^{2 / n+1}}+s \frac{1}{1-z^{n}}\right|>\left|s \frac{-z^{n-2}}{1-z^{n}}\right|=\left|g^{\prime}(z)\right|$. Since $z \in \Delta$ and $s \in \mathbb{R}$, we need $\left|\frac{(1-s)\left(1+z^{n}\right)}{\left(1-z^{n}\right)^{2 / n}}+s\right|>|s|$, or $\operatorname{Re}\left[\frac{1+z^{n}}{\left(1-z^{n}\right)^{2 / n}}\right]>0$. Letting $w=z^{n}$, for all $z \in \Delta$, we are left to showing that

$$
\operatorname{Re}\left[\frac{1+w}{(1-w)^{2 / n}}\right]>0 .
$$

Since $\operatorname{Re}(z)>0$ is equivalent to $|\operatorname{Arg}(z)|<\frac{\pi}{2}$, it suffices to show that

$$
\left|\operatorname{Arg}(1+w)-\frac{2}{n} \operatorname{Arg}(1-w)\right|<\frac{\pi}{2},
$$

where $\operatorname{Arg}(f)$ is the principal value of the argument of $f$. Now for $w \in \Delta,(1-w)$ and $(1+w)$ are points in the disk centered at 1 of radius 1 . Hence $\operatorname{Arg}(1-w)$ and 
$\operatorname{Arg}(1+w)$ are both between $\frac{-\pi}{2}$ and $\frac{\pi}{2}$. In fact, the line connecting the two points has 1 as its midpoint. Also $|\operatorname{Arg}(1+w)-\operatorname{Arg}(1-w)|<\frac{\pi}{2}$, and $\operatorname{Arg}(1+w)$ and $-\operatorname{Arg}(1-w)$ have the same sign. Further, $\frac{2}{n}|\operatorname{Arg}(1-w)| \leq|\operatorname{Arg}(1-w)|$. Using these facts we see that if $w \in \mathbb{R}$, then $\left|\operatorname{Arg}(1+w)-\frac{2}{n} \operatorname{Arg}(1-w)\right|=0$ and if $w \notin \mathbb{R}$, $\left|\operatorname{Arg}(1+w)-\frac{2}{n} \operatorname{Arg}(1-w)\right|<\frac{\pi}{2}$.

Lemma 2.3. Let $s$ be fixed such that $0 \leq s<1$. For any $n \geq 2, f$ is univalent in $\Delta$.

Proof. Fix $r_{0}$ such that $0<r_{0}<1$ and consider $\Omega \subset \Delta$ the region bounded by $\sigma_{1} \cup\{0\}, \sigma_{2}, \sigma_{3}$, and $\sigma_{4}$, where $\sigma_{1}=\left\{r: 0<r \leq r_{0}\right\}, \sigma_{2}=\left\{r e^{i \pi / n}: 0<r \leq 1\right\}$, $\sigma_{3}=\left\{e^{i \pi(1-r) / n}: 0 \leq r \leq r_{0}\right\}$, and $\sigma_{4}=\left\{z=t r_{0}+(1-t) e^{i \pi\left(1-r_{0}\right) / n}: 0 \leq t \leq 1\right\}$. We will prove this claim in three steps. First, we will show that $f$ is univalent in $\Omega$ for $r_{0}$ arbitrarily close to 1 , and that $0 \leq \operatorname{Arg}(f(\Omega)) \leq \frac{\pi}{n}$. Second, we verify that $f$ is univalent in the sector $\Omega \cup \Omega^{\prime}$, where $\Omega^{\prime}$ is the reflection of $\Omega$ across the real axis, and $\frac{-\pi}{n} \leq \operatorname{Arg}\left(f\left(\Omega \cup \Omega^{\prime}\right)\right) \leq \frac{\pi}{n}$. Finally, we will verify that $f$ is univalent in $\Delta$.

Step One: The argument principle for harmonic functions [3] is valid if $f$ is continous on $\bar{D}, f(z) \neq 0$ on $\partial D$, and $f$ has no singular zeros in $D$, where $D$ is a Jordan domain. Note $z_{0}$ is a singular point if $f$ is neither sense-preserving nor sense-reversing at $z_{0}$. Because of Lemma 2.2, we can use the argument principle. We will show that for arbitrary $M>0$, we may choose $r_{0}<1$ so that each value in the region bounded by $|w|<M$ and $0<\operatorname{Arg}(w)<\frac{\pi}{n}$ is assumed exactly once in the sector bounded by $|z|<1$ and $0<\operatorname{Arg}(z)<\frac{\pi}{n}$, while no value in the region bounded by $|w|<M$ and $\frac{\pi}{n}<\operatorname{Arg}(w)<2 \pi$ is assumed in this sector.

Observe that $f_{1}^{\prime}(z)=0$ only if $z$ is an $n$th root of -1 . Thus, on $\sigma_{1}, f_{1}$ is an increasing function of $r$ with $\operatorname{Arg}\left(f_{1}\right)=0$. Also, as $|z|$ increases on $\sigma_{2}$ and $\operatorname{Arg}(z)$ decreases on $\sigma_{3},\left|f_{1}(z)\right|$ increases. Note that $\operatorname{Arg}\left(f_{1}\left(\sigma_{2} \cup \sigma_{3}\right)\right)=\frac{\pi}{n}$. For $f_{2}$, if we let $z=\rho e^{i \theta}$ and use the fact that $f_{2}=h_{2}+\overline{g_{2}}$, we get

$$
\frac{\partial}{\partial \rho}\left(f_{2}\left(\rho e^{i \theta}\right)\right)=\frac{e^{i \theta}}{1-\rho^{n} e^{i n \theta}}+\overline{\frac{-\rho^{n-2} e^{i(n-1) \theta}}{1-\rho^{n} e^{i n \theta}}} .
$$

Note that $f_{2}(0)=0$. For $z \in \sigma_{1}$ and $n \geq 3, \frac{d}{d \rho}\left(f_{2}(\rho)\right)=\frac{1-\rho^{n-2}}{1-\rho^{n}}>0$, and so $f_{2}$ increases on $\sigma_{1}$ as $r$ increases. Also $f_{2}(\rho)>0$; hence $\operatorname{Arg}\left(f_{2}\left(\sigma_{1}\right)\right)=0$. (Recall that for $n=2, f_{2}\left(\sigma_{1}\right)=\{0\}$.) For $z \in \sigma_{2}$ and $n \geq 2, \frac{d}{d \rho}\left(f_{2}\left(\rho e^{i \pi / n}\right)\right)=e^{i \pi / n}\left(\frac{1+\rho^{n-2}}{1+\rho^{n}}\right) \neq$ 0 , and so $f_{2}\left(\sigma_{2}\right)$ does not reverse its direction. Further,

$$
f_{2}\left(\rho e^{i \pi / n}\right)=e^{i \pi / n}\left(\sum_{k=0}^{\infty} \frac{(-1)^{k} \rho^{k n+1}}{k n+1}-\sum_{k=1}^{\infty} \frac{(-1)^{k} \rho^{k n-1}}{k n-1}\right)=e^{i \pi / n} \tilde{\rho},
$$

where $\tilde{\rho} \in \mathbb{R}$; hence $\operatorname{Arg}\left(f_{2}\left(\sigma_{2}\right)\right)=\frac{\pi}{n}$. Recall $f_{2}$ is constant on $\sigma_{3}$. Therefore, we see that for $j=1,2,3, f\left(\sigma_{j}\right)$ is a simple curve with $\operatorname{Arg}\left(f\left(\sigma_{1}\right)\right)=0$ while $\operatorname{Arg}\left(f\left(\sigma_{2} \cup \sigma_{3}\right)\right)=\frac{\pi}{n}$. To complete the proof that $f$ is univalent on $\Omega$, it suffices to show that given any $M>0$ there exists an $r_{0}$ such that $|f(z)|>M$ for all $z \in \sigma_{4}$. To see this note that $\left|f_{2}(z)\right| \leq \pi /(n \sin (\pi / n))$ for all $z \in \Delta$ while for $s$ fixed $(0 \leq s<1)$ and for $z \in \sigma_{4},(1-s) f_{1}(z) \rightarrow \infty$ as $r \rightarrow 1$. Hence for a given $M$ the inequality will hold if we take $r_{0}$ sufficiently close to 1 . The proof is now complete since we have shown that every point outside the wedge is not assumed while every point inside the wedge is assumed exactly once by $f$. 
Step Two: Since $f$ is univalent in $\Omega$, we can use reflection across the real axis to establish that $f$ is univalent in the sector $\Omega^{\prime}$. In particular, suppose $z_{1}, z_{2} \in \Omega^{\prime}$ with $f\left(z_{1}\right)=f\left(z_{2}\right)$. Then by symmetry $\overline{f\left(\overline{z_{1}}\right)}=f\left(z_{1}\right)=f\left(z_{2}\right)=\overline{f\left(\overline{z_{2}}\right)}$. Hence, $f\left(\overline{z_{1}}\right)=f\left(\overline{z_{2}}\right)$, or $\overline{z_{1}}=\overline{z_{2}}$. Arguing in the same manner as in Step One, we can show that $0 \geq \operatorname{Arg}\left(f\left(\Omega^{\prime}\right)\right) \geq \frac{-\pi}{n}$. Therefore, $f$ is univalent in $\Omega \cup \Omega^{\prime}$ and its image is in the wedge between the angles $\frac{-\pi}{n}$ and $\frac{\pi}{n}$.

Step Three: First, it is true that $e^{i \pi 2 j / n} f\left(z e^{-i \pi 2 j / n}\right)=f(z)$, for all $z \in \Delta$ where $j=0,1, \ldots, n$. To see this note that

$$
e^{i \pi 2 j / n} f_{1}\left(z e^{-i \pi 2 j / n}\right)=e^{i \pi 2 j / n}\left[\frac{z e^{-i \pi 2 j / n}}{\left(1-z^{n} e^{-i \pi 2 j}\right)^{2 / n}}\right]=f_{1}(z) .
$$

Then by letting $u=t+2 \pi j / n$ and using the periodicity of $f_{2}$, we derive that

$$
\begin{aligned}
e^{i \pi 2 j / n} & f_{2}\left(z e^{-i \pi 2 j / n}\right) \\
& =\pi /(n \sin (\pi / n))\left(\frac{e^{i \pi / n}}{2 \pi}\right)\left[\sum_{k=0}^{n-1} e^{i \pi 2(k+j) / n} \int_{2 \pi(k+j+1) / n}^{2 \pi(k+j) / n} R e \frac{1+z e^{-i u}}{1-z e^{-1 u}} d u\right] \\
& =f_{2}(z) .
\end{aligned}
$$

Now, using this fact that $e^{i \pi 2 j / n} f\left(z e^{-i \pi 2 j / n}\right)=f(z)$, we see that if $z$ is any point in $\Delta$, it can be rotated so that it is in the sector $\Omega^{\prime}$, in which $f$ is univalent, and then rotated back by multiplying by the constant $e^{i \pi 2 j / n}$ and hence preserving univalency.

Remark. From the proof of Lemma 2.3 we see that $f(\Delta)$ is an $n$-slit domain with the slits lying on the line $r e^{i \pi j / n}$, for $j=1, \ldots, n-1$ and $1 \leq r<\infty$.

Theorem 2.4. For $n \geq 3, f \in S_{H}^{O}$. If $n=2$, then $f \in S_{H}$.

Proof. This follows from Lemmas 2.2, and 2.3, and the comment after Lemma 2.1.

\section{THE INNER MAPPING RADIUS AND $n$-SLIT MAPPINGS}

The function $f$ maps $\Delta$ onto the $n$-slit domain whose slits start at points symmetrically placed on the circle of radius $(1-s) 4^{-1 / n}+s \pi /(n \sin (\pi / n))(0 \leq s<1)$. Hence as the value of $s$ begins at 0 and increases, the slits start at a distance of $\left(\frac{1}{4}\right)^{\frac{1}{n}}$ and move away from the origin. When $s>\left(1-4^{-1 / n}\right) /\left[\pi /(n \sin (\pi / n))-4^{-1 / n}\right]$, the unit circle is completely contained in the image of $f$. For $n=2, f \in S_{H}$ and as $s \rightarrow 1, f(\Delta)$ will contain all the points in the disk whose radius approaches $\frac{\pi}{2} \approx 1.57$. Hall [5] showed that for $f \in S_{H}, f(\Delta)$ cannot contain any larger disk. Also, since the analytic function $F(z)=\frac{z}{1-z^{2}}$ maps $\Delta$ onto the 2 -slit domain whose slits start at $\pm \frac{i}{2}$, we have that $\tilde{F}(z)=(1-s+s \pi) \frac{z}{1-z^{2}}$, where $0 \leq s<1$, maps $\Delta$ onto $f(\Delta)$. Hence as $s$ approaches 1, the inner mapping radius of the harmonic 2-slit map $f$ approaches $\pi$. Thus $f$ provides a counterexample to Sheil-Small's conjecture [1, 6] that $\rho(f) \leq \frac{\pi}{2}$. For $n=3, f \in S_{H}^{O}$ and its image will contain all the points in the disk whose radius approaches $\frac{2 \pi \sqrt{3}}{9}<1.21$. Clunie and Sheil-Small [2] proved that $f(\Delta)$ cannot contain any larger disk. Our example shows that this number is sharp. We have not found a conjecture in the literature about the upper bound for the inner mapping radius of a function in $S_{H}^{O}$. Using the map $F(z)=\frac{2^{5 / 3} \pi \sqrt{3}}{9}\left(\frac{z}{\left(1-z^{3}\right)^{2 / 3}}\right)$, 
we see that for our 3 -slit map $f, \rho_{O}(f) \rightarrow \frac{2^{5 / 3} \pi \sqrt{3}}{9}>1.91$. For all of these $n$-slit mappings, where $n \geq 3$, the 3 -slit mapping gives the largest value for $\rho_{O}(f)$.

In conclusion, the author would like to thank T.J. Suffridge and the referee for their helpful suggestions.

\section{REFERENCES}

1. Bshouty, D. and W. Hengartner (editors), Problems and conjectures for harmonic mappings, from a workshop held at the Technion, Haifa, May 1995.

2. Clunie, J. and T. Sheil-Small, Harmonic univalent functions, Ann. Acad. Sci. Fenn. Ser. A.I Math. 9 (1984), 3-25. MR 85i:30014

3. Duren, P., W. Hengartner, and R. Laugesen, The argument principle for harmonic functions, Am. Math. Monthly 103 (1996), 411-415.

4. Duren, P., A survey of harmonic mappings in the plane, Texas Tech. Univ., Math. Series, Visiting Scholars Lectures, 1990-1992 18 (1992), 1-15.

5. Hall, R., A class of isoperimetric inequalities, J. Analyse Math. 45 (1985), 169-180. MR

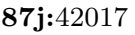

6. Sheil-Small, T., Constants for planar harmonic mappings, J. London Math. Soc. 42 (1990), 237-248. MR 91k:30052

7. MR 91b:30002

Department of Mathematics and Statistics, University of Missouri-Rolla, Rolla, Missouri 65409-0020

E-mail address: mdorff@umr.edu 